\title{
Cardiology day hospital: a 5-year experience
}

\section{(D)Renee Mixich ${ }^{*}$, DSenka Pejković}

University Hospital Dubrava, Zagreb, Croatia

\section{RECEIVED:}

September 7, 2021

ACCEPTED:

September 14, 2021

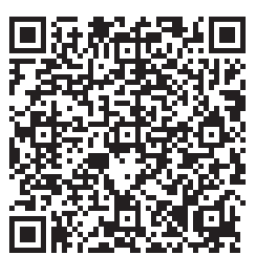

$\square$ Cardiologia Croatica 2021;16(11-12):362.
KEYWORDS: cardiology day hospital, nursing care, quality management.

CITATION: Cardiol Croat. 2021;16(11-12):362. | https://doi.org/10.15836/ccar2021.362

*ADDRESS FOR CORRESPONDENCE: Renee Mixich, Klinička bolnica Dubrava, Avenija Gojka Šuška 6, HR-10000 Zagreb, Croatia / Phone +385-1-290-2545 / E-mail: renee.mixich@gmail.com

ORCID: Renee Mixich, https://orcid.org/0000-0002-0991-7515 • Senka Pejković, https://orcid.org/0000-0002-7557-9358

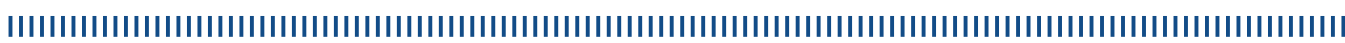

The cardiology day hospital (CDH) is an alternative for scheduled cardiac and in most cases diagnostic procedures. In the University Hospital Dubrava at the Department for Cardiovascular Medicine, the $\mathrm{CDH}$ allows many of the invasive procedures to be performed on an outpatient basis, while maintaining the quality of care. The aim of this short overview and experience at the University Hospital Dubrava was to perform a descriptive analysis of the activity carried out and to evaluate the quality of the provided care.

In a public-funded health system, it is essential to make the most of available resources. Clinical management encourages cost containment by avoiding unnecessary hospitalizations without decreasing the quality of the medical care provided. One approach to achieve this objective is to perform lowerrisk, elective, diagnostic and therapeutic invasive procedures. A daily hospitalization refers to a hospital stay lasting for several hours for various purposes: non-invasive diagnostic procedures, clinical and other examinations, and treatments that cannot be performed in the outpatient clinic but do not justify hospital admittance. It is a medical care unit where certain interventions are performed and post-procedure medical or nursing attention is provided under the supervision of a specialized medical team.

In the field of cardiovascular diseases, several diagnostic and therapeutic activities can be carried out during a short stay. A very common procedure that can be performed in this way is cardiac catheterization via radial artery access, as it reduces the incidence of complications and simplifies puncture site care in both diagnostic and interventional procedures. Heart failure is one of the most prevalent cardiovascular syndromes, whose treatment makes the most of $\mathrm{CDH}$ resources. ${ }^{1} \mathrm{CDH}$ units have proven effective in the treatment of these patients and have reduced the number of emergency room visits and rehospitalizations. Day hospitals are excellent support, as they facilitate close follow-up after hospital discharge and offer patients fast access to care in situations of clinical worsening.

This benefit could be extended to other patients with a prompt hospital discharge or patients seen in the emergency department (e.g., pericardial syndromes, atrial fibrillation, following heart surgery), who might need preferential access to reassessment, diagnostic tests, or therapeutic interventions that cannot be covered in the outpatient setting. The cardiology day is conceived as an area designed to provide complex or sophisticated medical care with little delay and avoiding hospitalization in patients with cardiovascular conditions that can be treated in an ambulatory setting.

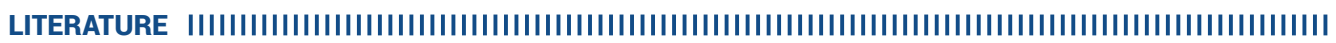

1. Udovičić M, Jakš̌ić Jurinjak S, Ivanović Mihajlović V, Falak H, Starčević B. Heart failure hospitalizations in University Hospital Dubrava 2007-2016. Cardiol Croat. 2018:13(11-12):376. https://doi.org/10.15836/ccar2018.376 